\title{
Protocol for tridimensional analysis of premaxila and nasopalatine canal using Cone-Beam Computed Tomography
}

\section{ANA FERNÁNDEZ-ALONSO ( $\sim$ annickfdzal@gmail.com )}

Dentistry Research Group

MARIA MERCEDES SUAREZ-CUNQUEIRO ( $\sim$ mariamercedes.suarez@usc.es )

Dentistry Research Group and Health Research Institute of Santiago (IDIS); Santiago de Compostela, Spain.

JUAN MUINELO-LORENZO

Dentistry Research Group

JUAN CARLOS PÉREZ-VARELA

Dentistry Research Group

\section{Method Article}

Keywords: cone-beam computed tomography, nasopalatine canal, dental implant, oral surgery, 3D analysis

Posted Date: November 20th, 2017

DOI: https://doi.org/10.1038/protex.2017.127

License: (c) (1) This work is licensed under a Creative Commons Attribution 4.0 International License. Read Full License 


\section{Abstract}

We provide a protocol for the analysis of nasopalatine canal $\backslash(\mathrm{NC})$ and the premaxilla using cone beam computed tomography. Bone ridge resorption and NC morphology must be taken into consideration for adequate treatment planning for dental surgery, such as dental implant placement. This protocol is used to evaluate at the three anatomic planes $\backslash$ (sagittal, coronal and axial) the dimensions of $\mathrm{NC}$, buccal bone plate and palatal bone plate. The protocol establish three levels above the NC to measure anatomic structures: level 1 , when the incisive foramen is completely closed on the axial plane; level 2, at the middle point of the NC length on the sagittal plane; and level 3, at the foramina of Stenson on the sagittal plane.

\section{Introduction}

**Development of the protocol** This protocol has been development to standardize the threedimensional analysis of the nasopalatine canal $\backslash(\mathrm{NC})$ and premaxilla so that future researchers will be able to obtain verifiable results for a variety of populations. This protocol is used to standardize conebeam computed tomography $\backslash(\mathrm{CBCT})$ analysis using all three anatomical planes. Despite using a radiological test that provides three-dimensional information of anatomical structures, most published CBCT studies currently available involve analysis on only one or two anatomic planes. ${ }^{*}$ Applications of the method ${ }^{\star \star}$ This protocol is valid for premaxilla assessment prior to placement of dental implants. One part of the protocol can be used for studying the crest in other anatomical areas of the jaw. It is possible to evaluate crestal dimensions on the three anatomical planes and at three levels, thus, gaining information regarding real volume in addition to observing how resorption affects the whole ridge, from coronal to apical of alveolar ridge. ${ }^{* *}$ Comparison with other methods ${ }^{* *}$ We have noted in the scientific literature that there is a lack of standardization regarding the measurement of dimensions. ${ }^{1-3}$ For example, the study by Tözüm et al. ${ }^{1}$ regarding palatal bone plate \(PBP) widths, established different benchmarks from the present study. ${ }^{4}$ The PBP width in the coronal area established by these authors ${ }^{1}$ is equivalent to our $\mathrm{PW}^{1.4}$ However, their study ${ }^{1}$ does not mention where the posterior limit of the apical width is set. The width they define as "apical palatal width" is measured from the most vestibular and apical point of the PBP to the most posterior point of the PBP, which they do not define. We believe that defining this point is vital to standardization, and it should extend the entire length of the horizontal plate of palatine bone. Our study ${ }^{4}$ sets the most posterior point of the PBP at the maxilla-palatine bone union, ie, the transverse palatine suture. With respect to medial PBP width, these authors ${ }^{1}$ set it at the midpoint of the PBP length. The advantage of using our CBCT protocol is that it provides morphological and dimensional information in the three anatomical planes, thus, giving the clinician complete volumetric information of the surgical treatment area. Previous studies do not make full use of CBCT information. ${ }^{3,5,6}$ For example, Bornstein et al. ${ }^{5}$ studied NC morphology in the coronal plane and alveolar ridge dimensions in the sagittal plane. Mardinger et al. ${ }^{3}$ evaluated only a few NC dimensions and the adjacent alveolar ridge, making it impossible to make associations between age, morphology and ridge resorption that could be useful for assessing potential bone regeneration prior to dental implants. In general, little literature is available regarding the axial plane. Nevertheless, as our research group has 
previously reported, ${ }^{7}$ the axial plane provides important information that cannot be obtained using only the sagittal and coronal CBCT planes, such as the distinction between up to 10 types of NC based on the number of internal canaliculi. The periodontal state variable was included in the present protocol because, beyond dental status, it is also important to know the periodontal condition of central incisors due to their proximity to NC. To the best of our knowledge, there are no previous studies evaluating periodontal condition as it relates to $\mathrm{NC}$ morphometry. However, several studies assess periodontal bone defects in the $\mathrm{CBCT}^{8-10}$ The study by Feijo et al. ${ }^{10}$ analyzed horizontal bone loss by comparing clinical outcomes with $\mathrm{CBCT}$ measures, and verified the accuracy of СВCT because no statistically significant differences were found. Fleiner et al. ${ }^{8}$ quantified periodontal bone loss in circumferential defects, comparing CBCT with direct measurement in human skulls. Other studies $9,11,12$ confirmed the accuracy of $\mathrm{CBCT}$ for detecting periodontal bone defects as results were similar to those obtained with intraoral radiographs and clinical periodontal diagnosis. Several studies consider CBCT to be more accurate for the morphological description of periodontal bone defects, while intraoral periodontal radiography provides better bone details. ${ }^{13,14}$ However, we should remember that the СВCT 3 dimensional image also provides information regarding vestibular and lingual/ palatal bone defects that $2 \mathrm{D}$ images from intraoral periodontal radiographies cannot provide. Regarding image quality, several studies $9,15-17$ indicate that a voxel size of less than $0.3 \mathrm{~mm}$ is necessary for adequate visibility of periodontal structures, as was have done in the present protocol. ${ }^{*}$ Experimental Design ${ }^{\star \star}$ The present CBCT protocol can be used in clinical practice and research to conduct a thorough study of patients who need dental surgery, implants or orthodontic treatment. It can be used with all types of CBCT equipment because the landmarks that we have set are not altered. ${ }^{*}$ Level of expertise needed to implement the protocol ${ }^{\star \star} A$ trained graduate student could apply this protocol, and then, an intra and interobserver evaluation should be carried out. The examiner should also be familiar with the interpretation of radiological images. ${ }^{*}$ Limitations ${ }^{\star *}$ This protocol is based solely on CBCT radiological data. No complementary clinical protocol has been designed, which would be helpful regarding such information as ethnicity, patient history prior to surgery, syndromes or clinical periodontal parameters.

\section{Reagents}

This protocol can be applied with any CBCT equipment.

\section{Equipment}

i-CAT® Model 17-19 \(Imaging Sciences International, Inc., Hatfield, Pennsylvania, USA) with a flat-panel detector of amorphous silicon, and an exposure protocol of $120 \mathrm{kVp}$, a current of $5 \mathrm{~mA}, 14.7 \mathrm{~s}$. The occlusal plane of patients was set parallel to the floor base by means of ear rods and a chin rest. DICOM files were reconstructed on computer $\backslash$ (Samsung R522, Samsung Electronics, South Korea) using the 3D visualization and measurement of iCATVision software \(i-CATVision 1.9, Imaging Sciences International, Inc., Hatfield, Pennsylvania, USA). To optimize image quality, a voxel size of 0.3 or less and a slice thickness of $0.25 \mathrm{~mm}$ should be used. For greater consistency, the inclusion criteria for the sample should 
be: 1) patients without defects in premaxilla area; and the exclusion criteria should be: 1) impacted teeth in the anatomical area of interest, 2) presence of a radiolucent or radiopaque lesion, 3) root fragments present, 4) dental implants in the region of interest, 5) suspected NC pathology $\backslash$ (cyst), 6) bone grafts, and 7) presence of orthodontic expanders.

\section{Procedure}

**Performing of CBCT: patient placement ${ }^{\star *}$ The ray technician places the patient sitting in the i-CAT unit with the head positioned between the beam and the receiver. Adjust the height of the patient until the chin lies on the chin rest, remaining in an upright posture, and also adjust the headband around the forehead. The correct position is marked by laser lines projected onto the patient's head: a horizontal line coinciding with the occlusal plane, a vertical line $3.5 \mathrm{~cm}$ in front of the condyle, and another vertical line cutting across the sagittal plane of the patient's head. Perform a preview and scan of the patient's head. In adult patients use a radiation dose of $120 \mathrm{Kv}$ and $5 \mathrm{~mA}$, a radiation time of 14.7 seconds, a focus-plate distance of $70 \mathrm{~cm}$, a resolution of 0.25 or 0.3 voxels and a field of view from 70 to $110 \mathrm{~cm}$. Once the scanner has been completed, reconstruct digitally. ${ }^{*}$ Choice and evaluation of the image ${ }^{\star \star}$ Analyze images with the i-CATVision program in the MPR Screen format, and simultaneously perform an analysis of the axial, sagittal and coronal anatomical planes. Arrange all cursors with a cut-off amplitude of 0.25 $\mathrm{mm}$. Perform linear and angular measurements of $\mathrm{NC}$, as well as buccal bone plate $\backslash(\mathrm{BBP})$ and palatal bone plate $\backslash(\mathrm{PBP})$ on the three anatomical planes. In addition, perform a morphological analysis on the three anatomical planes of NC. Image evaluation: NC and bone plates measurements Make NC, the BBP and PBP measurements at three locations along the NC that we define as: level 1, level 2 and level 3. Do this simultaneously for all three anatomical planes $\backslash$ (axial, sagittal and coronal) as follows $\backslash$ (Figure 1 ). i. Level 1 To find level 1, go to axial slice and find the NC image. To standardize our analysis, we must locate the axial slice when the incisive foramen of $N C$ is completely closed $\backslash$ (Figure 2 and 3). For this, perform a dynamic vision in axial slice, i.e., go to the most coronal zone of $\mathrm{NC}$, and visualize the sequence of radiographies from the oral cavity to the nasal cavity, to visualize the closed NC at incisive foramen or level 1 . We recommend performing measurements when level 1 is located in the three anatomic planes to optimize working time, as you move the cursor in the axial plane, the cursors of the sagittal and coronal planes are taken automatically to their respective level 1 's $\backslash$ (Figure 3). In axial slice, take the following measurements. The diameters of $\mathrm{NC}$ are: buccal-palatal diameter $\backslash(\mathrm{bpD} 1)$ and transversal diameter $\backslash(\mathrm{tD} 1)$ of NC. The bpD1 corresponds to maximum diameter between buccal and palatal bone cortical of NC; the tD1 is the maximum diameter perpendicular to bpD1 \(Figure 4). The BBP widths are the distances from BBP buccal wall to NC at three points of NC: left $\backslash($ LW1), central $\backslash(C W 1)$ and right $\backslash(\mathrm{RW} 1)$. LW1 corresponds to the width that is established between the vestibular cortical of NC left border and the BBP external plate that is anterior to NC. CW1 corresponds to the width that is established between the anterior nasal spine and the most anterior and central point of the NC. RW1 corresponds to the width that is established between vestibular cortical of NC right border and the BBP external plate that is anterior to $\mathrm{NC} \backslash$ (Figure 5). In coronal slice, take the following measurement on the horizontal line: NC diameter at level $1 \backslash(\mathrm{CD} 1) \backslash($ Figure 6). In sagittal slice, take the following 
measurements on the horizontal line: BBP width at level $1 \backslash($ BW1), NC sagittal diameter at level $1 \backslash(\mathrm{SD} 1)$, and PBP width at level $1 \backslash(P W 1)$. In addition, take the following measurements from the horizontal line to the inferior edges of BBP and PBP: BBP length at level $1 \backslash(B L 1)$, and PBP length at level $1 \backslash(P L 1) \backslash($ Figure 7). Figure 8 shows the simultaneous measurements made for the three anatomical planes. ii. Level 2 To locate the level 2, you must first measure the length of $\mathrm{NC} \backslash(\mathrm{NCL})$, which corresponds to the distance between the incisive foramen and foramina of Stenson in the sagittal slice \(Figure 9). To find level 2, place the red horizontal line at the middle point NCL in the sagittal plane. \(Figure 9). In axial slice, take the following measurements: buccal-palatal diameter $\backslash(\mathrm{bpD} 2)$ and transversal diameter $\backslash(\mathrm{tD} 2)$ of NC. The bpD2 corresponds to maximum diameter between buccal and palatal bone cortical of NC; the tD2 is the maximum diameter perpendicular to bpD2 \(Figure 10). BBP widths are the distances from BBP buccal wall of NC at three points of NC: left $\backslash(L W 2)$, central $\backslash(C W 2)$ and right $\backslash(R W 2) \backslash$ (Figure 11). LW2 corresponds to the width that is established between the vestibular cortical of $\mathrm{NC}$ left border and the BBP external plate that is anterior to NC. CW2 corresponds to the width that is established between the anterior nasal spine and the most anterior and central point of the NC. RW2 corresponds to the width that is established between vestibular cortical of NC right border and the BBP external plate that is anterior to NC \(Figure 11). In coronal slice, take the following measurement on the horizontal line: NC diameter at level $2 \backslash(C D 2) \backslash$ (Figure 12). In sagittal slice, take the following measurements on the horizontal line: BBP width at level $2 \backslash(B W 2), N C$ sagittal diameter at level $2 \backslash($ SD2), and PBP width at level $2 \backslash(P W 2)$. In addition, take the following measurements from the horizontal line to the inferior edges of BBP and PBP: BBP length at level $2 \backslash(B L 2)$, and PBP length at level $2 \backslash(P L 2) \backslash($ Figure 13). Figure 14 shows the simultaneous measurements made for the three anatomical planes. iii. Level 3 To find level 3 , move the red horizontal line to the foramina of Stenson on sagittal plane. Perform measurements for the coronal and sagittal planes, excluding the axial plane because of poor image quality at the level of foramina of Stenson. In coronal slice, take the following measurement on the horizontal line: NC diameter at level $3 \backslash$ (CD3) $\backslash$ (Figure 15). In sagittal slice, take the following measurements on the horizontal line: PBP width at level $3 \backslash(P W 3)$, this is the width from the most posterior point of the transverse palatine suture $\backslash$ (Figure16) to the palatal wall of NC, NC sagittal diameter at level $3 \backslash($ SD3), and PBP width at level $3 \backslash$ (PW3). In addition, take the following measurements from the horizontal line to the inferior edges of BBP and PBP: BBP length at level $3 \backslash(\mathrm{BL} 3)$. PBP length at level $3 \backslash(\mathrm{PL} 3)$, and, the total BBP length $\backslash(\mathrm{tBL})$ is also calculated by joining midpoints of coronal and apical BBP width $\backslash$ (Figure 17). Figure 18 shows the simultaneous measurements made for the coronal and sagittal planes. We can observe the poor image quality of axial plane at foramina of Stenson level. iv. Nasopalatine angle To obtain the nasopalatine angle, draw the LNC and the tangential line to the nasal floor. After this, simply measure the angle formed between these lines $\backslash$ (Figure 19). v. Morfological classification of NC Classify NC morphology in each anatomic plane, following the classification by Mardinger et al.3 and Song et al.18 for the sagittal plane, the classification by Bornstein et al. 5 for the coronal plane and the classification established by our research team for the axial plane.7 In order to establish NC morphology, evaluate all the radiological slices pertaining to NC. To do so in the sagittal plane, evaluate all the sagittal NC images; in the coronal plane evalute images from anterior to posterior; and for the axial plane evaluate from the incisive foramen to the foramina of Stenson. Classify NC morphology in sagittal slice according to the protocol 
established by Mardinger et al.,3 who typified the NC into the following four forms: 1) Funnel: NCs with a narrower apical anatomy that widens coronally $\backslash($ Figure 20a and 21a). 2) Cylindrical: NCs with a stable morphology, ie, the NC presents both parallel vestibular and palatal cortices from the incisor foramen to the foramina of Stenson $\backslash$ (Figure 20b and 21b). 3) Hourglass: NCs with both a wide incisor foramen and a wide foramina of Stenson, and a narrow area in the medial zone \(Figure 20c and 21c). 4) Half-moon $\backslash$ (or banana): NCs with a wider area in the medial zone \(Figure 20d and 21d). To establish the direction and course of the NC in sagittal slice, follow the protocol by Song et al.18 The direction of the NC is considered vertical when the canal runs perpendicular from the nasal floor to the oral cavity; and it is considered oblique when the trajectory is not perpendicular. The NC course is considered straight for canals that communicate the foramina of Stenson and the incisive foramen without linear breaks; and, otherwise is considered to be curved. We distinguish four categories $\backslash($ Figure 22 and 23): 1) Verticalstraight $\backslash($ la). 2) Vertical-curved $\backslash(\mathrm{Ib})$. 3) Oblique-straight $\backslash(\mathrm{Ila})$. 4) Oblique-curved $\backslash(\mathrm{Ilb})$. Evaluate the NC coronal morphology by checking all tomographic slices containing the NC from anterior to posterior. Then classify into three main categories according to the classification by Bornstein et al.5 (Figure 24 and 25): 1) Single. All slices show a single cylindrical canal $\backslash($ Figure 24a and 25a). 2) Separate parallels. All slices show two seperate parallel canals $\backslash$ (Figure 24b and 25b). 3) Y-type $\backslash(\mathrm{Ya}, \mathrm{Yb}, \mathrm{Yc})$. All slices show a $\mathrm{Y}$ shaped canal, i.e., two branches that descend from the foramina of Stenson that anastomose into one incisive foramen. There are three subcategories: i. Ya. Two definite branches descending from the foramina of Stenson $\backslash$ (Figure 24c and 25c). ii. Yb. One of the two branches descending from the foramina of Stenson is bifurcated $\backslash$ (Figure $24 \mathrm{~d}$ and $25 \mathrm{~d}$ ). iii. Yc. The two branches descending from the Stenson's foramina are bifurcated $\backslash$ (Figure $24 \mathrm{e}$ and $25 \mathrm{e}$ ). If you observe more than one NC, as in Figure 26 , use our team's 7 modification of the classification by Bornstein et al. 5 Figure 27 presents sample combinations of NC coronal morphology. In the axial plane, classify NC by number of incisive foramina in level 1, and number of foramina of Stenson in level 3, as established by our research team.4 Make a designation with two figures $\backslash(" X-X ")$, where the first refers to the number of incisive foramina, and the second refers to the number of foramina of Stenson \(Figure 28). **Periodontal condition of the remaining teeth ${ }^{\star *}$ The periodontal condition of remaining teeth is important to keep in mind because this can modify the biological behavior of the alveolar bone. To evaluate periodontal bone loss on sagittal plane, measure the distance from the cemento-enamel junction to BBP in buccal surface and from the cemento-enamel junction to PBP in palate surface of teeth 11 and $21 \backslash$ (Figure 10). Periodontal condition is defined as the mean value of these measurements for both teeth 11 and 21 . Subjects are then classified into three groups. The first group is normal periodontal condition $\leq 3 \mathrm{~mm}, \backslash$ (based on Faria Vasconcelos et al.9). The other groups reflect different levels of periodontitis: moderate periodontitis $\backslash(>3-$ $\leq 6 \mathrm{~mm})$ and severe periodontitis $\backslash(>6 \mathrm{~mm}) \backslash($ Table 1$)$.

\section{Timing}

A trained examiner can carry out the entire protocol in 7 minutes per one CBCT analysis. The quality of the process is not affected by the time it takes. 


\section{Troubleshooting}

In case of difficulty visualizing anatomic structures, we recommend performing a dynamic display of the duct in the three anatomical planes $\backslash$ (sagittal, coronal and axial). In the sagittal plane, images should be viewed dynamically from left to right; in the coronal plane, images should be viewed posterior to anterior; and in the axial plane, images should be view from the top down $\backslash$ (video1). We can observe in the following videos the dynamic view of the $\mathrm{NC}$ in the three planes. In the video 2 we observed a patient with a combination of coronal shapes travelling from the anterior to the posterior slices of NC. In the most anterior zone of $\mathrm{NC}$ we observe a single canal and in posterior zone two canals. In the video 3 we observed a single anterior canal that is anastomosed with another single posterior canal. In video 4 , we can observe a computer simulation of $\mathrm{NC}$ with a combination of one single anterior that is anastomosed with an Y-type posterior canal.

\section{Anticipated Results}

The typical image that we can see is the $\mathrm{NC}$ as a radiolucent well-defined structure with different shapes.3,5-7 It is positioned in the center of the premaxilla, occupying approximately one-third of all premaxila \(Figure 11a). When problems exist for visualizing the NC may be due to artifacts caused by dental implants $\backslash$ (Figure 11b), to own pathology of NC such a cyst $\backslash$ (Figure 11c) or tumoral or cystic pathology in the adjacent bone. In these cases this protocol to standard measurements that we have proposed it is not possible to apply.

\section{References}

1 Tözüm, T. F. et al. Evaluation of maxillary incisive canal characteristics related to dental implant treatment with computerized tomography: a clinical multicenter study. J.Periodontol. 83, 337-343, doi:10.1902/jop.2011.110326 \(2012). 2 Thakur, A. R., Burde, K., Guttal, K. \& Naikmasur, V. G. Anatomy and morphology of the nasopalatine canal using cone-beam computed tomography. Imaging. Sci. Dent. 43, 273-281, doi:10.5624/isd.2013.43.4.273 \(2013). 3 Mardinger, O., Namani-Sadan, N., Chaushu, G. \& Schwartz-Arad, D. Morphologic changes of the nasopalatine canal related to dental implantation: a radiologic study in different degrees of absorbed maxillae. J. Periodontol. 79, 1659-1662, doi:10.1902/jop.2008.080043 \(2008). 4 Fernández-Alonso, A. et al. Critical anatomic region of nasopalatine canal based on tridimensional analysis: cone beam computed tomography. Sci. Rep. 5, 12568, doi:10.1038/srep12568 \(2015). 5 Bornstein, M. M., Balsiger, R., Sendi, P. \& von Arx, T. Morphology of the nasopalatine canal and dental implant surgery: a radiographic analysis of 100 consecutive patients using limited cone-beam computed tomography. Clin. Oral Implants Res. 22, 295-301, doi:10.1111/j.1600-0501.2010.02010.x \(2011). 6 Lopez Jornet, P., Boix, P., Sanchez Perez, A. \& Boracchia, A. Morphological Characterization of the Anterior Palatine Region Using Cone Beam Computed Tomography. Clin. Implant Dent. Relat. Res. 17, 459-64, doi:10.1111/cid.12271 \(2015). 7 Fernández-Alonso, A. et al. Three-dimensional study of nasopalatine canal morphology: a descriptive retrospective analysis using cone-beam computed tomography. Surg. Radiol. Anat. 36, 895-905, 
doi:10.1007/s00276-014-1297-3 \(2014). 8 Fleiner, J., Hannig, C., Schulze, D., Stricker, A. \& Jacobs, R. Digital method for quantification of circumferential periodontal bone level using cone beam CT. Clin. Oral Investig. 17, 389-396, doi:10.1007/s00784-012-0715-3 \(2013). 9 de Faria Vasconcelos, K. et al. Detection of periodontal bone loss using cone beam CT and intraoral radiography. Dentomaxillofac. Radiol. 41, 6469, doi:10.1259/dmfr/13676777 \(2012). 10 Feijo, C. V., Lucena, J. G., Kurita, L. M. \& Pereira, S. L. Evaluation of cone beam computed tomography in the detection of horizontal periodontal bone defects: an in vivo study. Int. J. Periodontics Restorative Dent. 32, e162-168 \(2012). 11 Misch, K. A., Yi, E. S. \& Sarment, D. P. Accuracy of cone beam computed tomography for periodontal defect measurements. J. Periodontol. 77, 1261-1266, doi:10.1902/jop.2006.050367 \(2006). 12 Braun, X., Ritter, L., Jervoe-Storm, P. M. \& Frentzen, M. Diagnostic accuracy of CBCT for periodontal lesions. Clin. Oral Investig. 18, 1229-1236, doi:10.1007/s00784-013-1106-0 \(2014). 13 Vandenberghe, B., Jacobs, R. \& Yang, J. Detection of periodontal bone loss using digital intraoral and cone beam computed tomography images: an in vitro assessment of bony and/or infrabony defects. Dentomaxillofac. Radiol. 37, 252-260, doi:10.1259/dmfr/57711133 \(2008). 14 Vandenberghe, B., Jacobs, R. \& Yang, J. Diagnostic validity $\backslash($ or acuity) of 2D CCD versus 3D CBCT-images for assessing periodontal breakdown. Oral Surg. Oral Med. Oral Pathol. Oral Radiol. Endod. 104, 395-401, doi:10.1016/j.tripleo.2007.03.012 \(2007). 15 Naitoh, M., Hirukawa, A., Katsumata, A. \& Ariji, E. Evaluation of voxel values in mandibular cancellous bone: relationship between cone-beam computed tomography and multislice helical computed tomography. Clin. Oral Implants Res. 20, 503-506, doi:10.1111/j.1600-0501.2008.01672.x \(2009). 16 Katsumata, A. et al. Relationship between density variability and imaging volume size in cone-beam computerized tomographic scanning of the maxillofacial region: an in vitro study. Oral Surg. Oral Med. Oral Pathol. Oral Radiol. Endod. 107, 420-425, doi:10.1016/j.tripleo.2008.05.049 \(2009). 17 Grimard, B. A. et al. Comparison of clinical, periapical radiograph, and cone-beam volume tomography measurement techniques for assessing bone level changes following regenerative periodontal therapy. J. Periodontol. 80, 48-55, doi:10.1902/jop.2009.080289 \(2009). 18 Song, W. C. et al. Microanatomy of the incisive canal using three-dimensional reconstruction of microCT images: an ex vivo study. Oral Surg. Oral Med. Oral Pathol. Oral Radiol. Endod. 108, 583-590, doi:10.1016/j.tripleo.2009.06.036\(2009). 19 Amler, M. H. The time sequence of tissue regeneration in human extraction wounds. Oral Surg. Oral Med. Oral Pathol. 27, 309-318 \(1969).

\section{Acknowledgements}

The authors express their gratitude to the radiologist Lilian Sampedro Crujeiras, for her exceptional work in the Radiology Unit.

\section{Figures}

Figure 1 
Analysis levels of NC Sagittal tomographic slice of NC with level distributions.

\section{Figure 2}

Axial level 1 Axial tomographic slice with incisive foramen of NC completely closed.

\section{Figure 3}

Level 1: three anatomic slices Simultaneous view of NC levels 1 in axial, sagittal and coronal slices.

\section{Figure 4}

Axial measurements Radiographies and draw-scheme of level 1. Axial slice: (1) bpD1, (2) tD1.

\section{Figure 5}

Axial bone mesurements Buccal bone width in axial slice at level 1. LW1: left width, CW1: central width and RW1: right width at level 1.

\section{Figure 6}

Coronal level 1 Coronal slice. CD1: coronal diameter at level 1.

原

\section{Figure 7}

Fiuge 7 Sagital level 1 measurements Sagittal slices. SD1: sagittal diameter at level 1, PW1/BW1: palatal /buccal width at level 1 and PL1/BL1: palatal/buccal length at level 1.

原

\section{Figure 8}

Simultaneous measurements of level 1 Three simultaneous radiographies at level 1. (a) Axial slice: (1) transversal diameter, (2) buccal-palatal diameter, (3-4-5) left-central-right width of buccal bone plate. (b) Sagittal slice: (1) palatal width, (2) sagittal diameter, (3) buccal width, (4) palatal length and (5) buccal length. (c) Coronal slice: coronal diameter.

Figure 9 
Location of level 2 Level 2: (a) axial slice, (b) sagittal slice: (1) incisive foramen, (2) foramina of Stenson, (3) nasopalatine canal length ( $\mathrm{NCL}$ ) and (4) mid-point of nasopalatine canal length. (c). Coronal slice.

\section{Figure 10}

Axial level 2 Axial slice at level 2: bpD2: buccal-palatal diameter, tD2: transversal diameter.

\section{Figure 11}

Axial level 2: bone measurements Axial slice at level 2: LW2/ CW2/ RW2: left-central-right width of buccal bone plate.

政

Figure 12

Coronal level 2 Coronal slice: CD2.

展

Figure 13

Sagittal level 2 Sagittal slices. SD2: sagittal diameter at level 2, PW2/BW2: palatal /buccal width at level 2 and PL2/BL2: palatal/buccal length at level 2.

\section{Figure 14}

Simultaneous measurements of level 2 Three simultaneous radiographies at level 2. (a) Axial slice: (1) transversal diameter, (2) buccal-palatal diameter, (3-4-5) left-central-right width of buccal bone plate. (b) Sagittal slice: (1) NC diameter in coronal region, (2) coronal diameter at Stenson'foramina, (3) NC length, (4) middle of NC length, (5) palatal width, (6) sagittal diameter, (7) buccal width, (8) palatal length and (9) buccal length. (c) Coronal slice: coronal diameter.

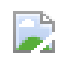

\section{Figure 15}

Coronal level 3 Coronal slice: CD3: coronal diameter at level 3.

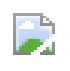

\section{Figure 16}

Transverse palatine suture Sagittal slice: the most posterior point of the transverse palatine suture. 


\section{Figure 17}

Sagittal level 3 Sagittal slice at level 3: (1) the most posterior point of the transverse palatine suture, PW3: palatal width, SD3: sagittal diameter, BW3: buccal width, PL3: palatal length and BL3: buccal length.

\section{Figure 18}

Simultaneous measurements of level 3 Level 3: (a) Axial slice. (b) Sagittal slice: (1) the most posterior point of the transverse palatine suture, (2) PW3, (3) SD3, (4) BW3, (5) PL3, (6) BL3 and (7) tBL. (b) Coronal slice: CD3.

娄

\section{Figure 19}

NC angle Nasopalatine angle: tangent to nasal floor (blue line), NC length (green line) and nasopalatine angle between anterior items (orange line).

\section{Figure 20}

Sagittal morphology Morphological sagittal shape classification of the nasopalatine canal. a) Funnel, b) cylindrical, c) hourglass, and d) banana-like.

\section{Figure 21}

Sagittal morphology in CBCT Sagittal radiographies with NC shapes: a) funnel, b) cylindrical, c) hourglass, and d) banana-like.

\section{Figure 22}

Direction-course Direction and course of nasopalatine canal. a) vertical-straight. b) vertical-curved. c) oblique-straight. d) oblique-curved.

\section{Figure 23}

Direction-couse in CBCT Sagittal radiographies with direction and course of nasopalatine canal: a) vertical-straight. b) vertical-curved. c) oblique-straight. d) oblique-curved. 


\section{Figure 24}

Coronal morphology Coronal morphology of nasopalatine canal: a) single, b) two separate parallel canals and variations of the Y-type canals: c) Ya, d) Yb and e) Yc.
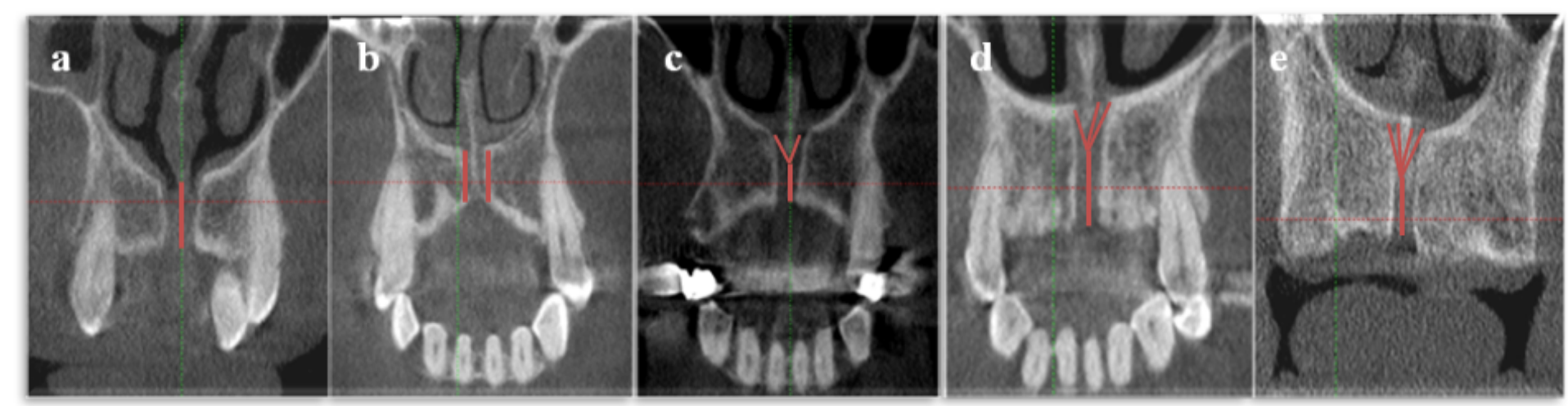

\section{Figure 25}

Coronal morphology in CBCT Coronal radiographies with coronal morphology of nasopalatine canal: a) single, b) two separate parallel canals and Y-type canals: c) Ya, d) Yb and e) Yc.

\section{Figure 26}

NC combination squeme Coronal morphology. We can observe a combination NC with an anterior single canal joining a Y-type canal. 
Figure 27

Coronal shapes combinations Combinations of different subgroups of coronal morphology. 1. A combination of (a) a single anterior canal and (b) two separate posterior canals. 2. A combination of (b) two anterior independent canals and (a) a single posterior canal. 3. A combination of (c) an anterior Ya type canal and (a) a single posterior canal. 4. A combination of (b) two anterior independent canals, (a) one single medial canal and one (a) single posterior canal.

\section{Figure 28}

Axial morphology Different axial categories. (a) single incisive foramen, (b) two incisive foramina, (c) three incisive foramina, (d) four incisive foramina and (e) five incisive foramina.

\section{Figure 29}

Periodontal measurements Sagittal slice. Periodontal bone loss in buccal (1) and palatine (2) surface of upper central incisor.

\section{Figure 30}

NC: differents states (a) Normal NC. (b) Dental implants cause problems for visualizing the NC. (c) NC cyst.

\section{Supplementary Files}

This is a list of supplementary files associated with this preprint. Click to download.

- supplement0.mov

- supplement0.mp4

- supplement0.mov

- supplement0.mov 